\title{
Role of low-latitude forests in modulating forest filter effect on a continental scale: Long-term simulation on PCB-153 in Chinese forests
}

\author{
Yue $\mathrm{Xu}^{\mathrm{a}, *}$, Chongguo Tian ${ }^{\mathrm{b}}$, Luca Nizzetto ${ }^{\mathrm{c}, \mathrm{d}}$, Gan Zhang ${ }^{\mathrm{e}}$ \\ a State Key Laboratory of Environmental Geochemistry, Institute of Geochemistry, Chinese Academy of Sciences, Guiyang 550002, China \\ ${ }^{\mathrm{b}}$ Key Laboratory of Coastal Zone Environmental Processes and Ecological Remediation, Yantai Institute of Coastal Zone Research, Chinese Academy of Sciences, Yantai 264003, China \\ ${ }^{c}$ Norwegian Institute for Water Research (NIVA), Gaustadalléen 21, 0349 Oslo, Norway \\ ${ }^{d}$ RECETOX, Masarik University, Kamenice 753/5, 62500 Brno, Czech Republic \\ e State Key Laboratory of Organic Geochemistry, Guangzhou Institute of Geochemistry, Chinese Academy of Sciences, Guangzhou 510640, China
}

\section{H I G H L I G H T S}

- A level IV fugacity model with a dynamicforest module was introduced.

- Low-latitude forests reduced the overall half-life of PCB-153 in China.

- The forest filter effect was achieved by enhanced degradation.

\section{A R T I C L E I N F O}

\section{Article history:}

Received 28 December 2020

Received in revised form 26 February 2021

Accepted 1 March 2021

Available online 8 March 2021

Editor: Jay Gan

\section{Keywords:}

Forest filter effect

Low-latitude forests

PCB-153 outflow reduction

Enhanced degradation

\section{GRAPHICA L A B S T R A C T}

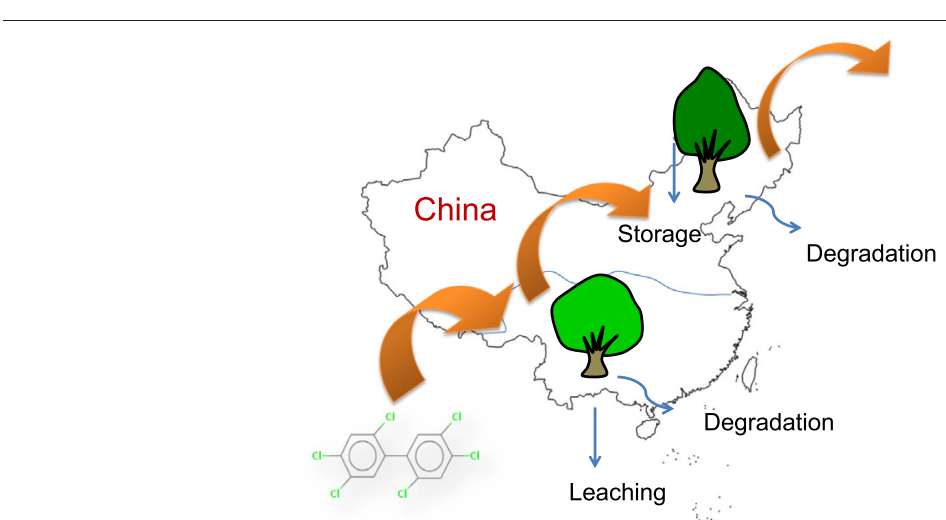

\begin{abstract}
A B S T R A C T
Forests are important compartments influencing the environmental fate of persistent organic pollutants (POPs). To illustrate the effect of forests on the regional cycle of POPs, a level IV fugacity fate and transport model coupled with a detailed dynamic-forest module was applied to simulate the long-term variations of PCB-153 in China, where forest coverage accounts for approximately one fifth of land area. In the scenarios with forests, atmospheric outflow from China was $69 \%$ of that in the scenario without forests due to the enhanced storage in soil, degradation, and leaching. Previous studies regarded high-latitude areas, such as the polar region and boreal forests, as environments capable of reducing mobility of PCB-153, and they act as sinks of POPs. This modeling result suggests that tropical and subtropical forests may also play a similar role despite high temperatures favoring volatilization. Unlike boreal forest, the low-latitude forests may reduce the overall lifetime of PCB-153 in China due to enhanced degradation in warmer and moist soils of the tropical and subtropical area. Given that approximately half of the global forests are located in tropical and subtropical regions, they can be important environments influencing the global geochemical cycle and distribution of POPs, hence deserving more scientific attention by modeling and empirical studies.
\end{abstract}

(c) 2021 Elsevier B.V. All rights reserved.

\footnotetext{
* Corresponding author.

E-mail address: xu-yue@mail.gyig.ac.cn (Y.Xu).
} 


\section{Introduction}

Forest ecosystem plays an important role on the global distribution of persistent organic pollutants (POPs), a group of toxic, persistent, and lipophilic pollutants. The "forest filter effect (FFE)" may significantly alter the environmental fate of POPs via increasing air-to-soil transport of POPs, reducing their long-range transport potential and influencing global fractionation (Moeckel et al., 2009). The interaction of POPs with forest system is controlled by physicochemical properties of these compounds, such as the octanol/air $\left(\mathrm{K}_{\mathrm{OA}}\right)$ and air/water $\left(\mathrm{K}_{\mathrm{AW}}\right)$ partition coefficients (Horstmann and McLachlan, 1998). With regard to compounds with $7<\log \mathrm{K}_{\mathrm{OA}}<11$ and $\log \mathrm{K}_{\mathrm{AW}}>6$, boreal forests can effectively facilitate their deposition from the air to the ground ( $\mathrm{Su}$ and Wania, 2005), delaying the atmospheric long-range transport. Moeckel et al. (2009) estimated that approximately 2-21\% of the global atmospheric emission of polychlorinated biphenyls (PCBs) enters boreal forests. Therefore, forest soils are likely to represent an underestimated sink of POPs (Dalla Valle et al., 2005).

Although those numerical estimations are useful in evaluating the FFEs, further assessments are hindered by poorly considered ecophysiological processes of plants, a more detailed description of the forest system functioning, and more accurate description of environmental parameters. The dynamics of vegetated systems, which might respond to the environmental variables in a nonlinear way, were previously simulated via simplified modeling approaches (Terzaghi et al., 2017). For example, leaves can partly buffer against variations of air temperature (Dong et al., 2017) due to evapotranspiration, requiring a physiological model to estimate POP partitioning between the air and leaf surface, However, the temperature differences between leaf and air were seldom considered in models of POPs, leading to uncertainties in the numerical simulation. Important processes, such as the fast leaching of PCBs in tropical forest (Zheng et al., 2015), bidirectional exchange between air and canopy (Nizzetto and Perlinger, 2012), and dynamic processes in litter (Terzaghi et al., 2017), were seldom considered in traditional regional models of POP fate. New models considering ecophysiological processes have been recently developed and applied to quantify air-canopy-litter-soil exchange (Nizzetto and Perlinger, 2012; Terzaghi et al., 2017). However, those simulations were conducted on the local scale over a relatively short time span. To evaluate the longterm FFE on a regional scale, a multimedia model considering the dynamics of chemical exchange between atmosphere and forests under realistic environmental conditions is still needed.

China is the country with the fastest growing forest area in the

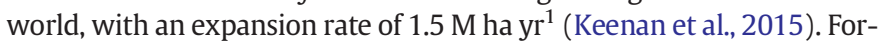
est coverage has reached $21 \%$ in the 2010 s, accounting for $49 \%$ of the national carbon pool (Tang et al., 2018). A wide variety of forest types, such as boreal, temperate, and subtropical and tropical biome, distributed over multiple climate regimes, representing a spatially-varying carbon pool of regional significance (Jiang et al., 1999). Although the Asian boreal forest only extends to the DaXing'anling Mountains in Northeast China, other forest biomes can also be a potential reservoir of POPs. A significant FFE for POPs has been assessed in Northeast and Southwest China and the Tibetan Plateau (Liu et al., 2014; Luo et al., 2019). Huang et al. (2016) estimated that the Three-Northern Region Shelter Forest in China can enhance the atmospheric removal of phenanthrene by $29 \%$ and benzo[a]pyrene by $53 \%$ compared with the simulation without the forest. Liu et al. (2017) calculated that the amounts of PCBs and PBDEs stored in the top $20 \mathrm{~cm}$ soil are 174 and $1410 \mathrm{t}$ in the planted forest, respectively. Recently, tropical forests have been studied in relation to the fast leaching of PCBs to deep soil due to rapid litter turnover and co-transport with dissolved organic carbon (Zheng et al., 2015). Tropical and subtropical forests in Southwest China had the highest carbon storage and density in the country (Qiu et al., 2020). Evidence suggests that low-latitude forests may contribute to the geochemical cycle of POPs. With the pronounced growth of those biomes in China, the storage capacity for POPs is expected to increase in the future, and represent an important regional sink of atmospheric POPs. However, the implication of increased sequestration of POPs by those forests for the accumulation and behavior of these contaminants in the terrestrial ecosystem is unclear.

In this study, a dynamic forest mathematical model is introduced considering plant physiology aspects and forest ecological processes and coupled on-line with a Gridded Pesticide Emission and Residue Model (GhnPERM) applied to the Chinese scenario. This tool was used to examine the responses of chemical transport and transfer to ecological processes. The model ultimately provides assessment of the mass balance of POPs in a spatially explicit multi-compartment regional environment. GhnPERM has been previously used to explore the environmental fate of POPs, such as $\alpha-\mathrm{HCH}$ and $\beta-\mathrm{HCH}$ (Xu et al., 2012; Xu et al., 2013) in China. PCB-153 was selected for this study because it falls within the "window" of partitioning property combinations expected to yield a significant FFE (Su and Wania, 2005). Such a numerical model was used as an example to illustrate the importance of forests on the environmental fate of PCB-153 in Mainland China. The PCB-153 overall mass balance between degradation, leaching, residue, and atmospheric secondary emissions with and without forest compartments was compared. We considered atmospheric inflow and outflow as the PCB-153 masses transported in to and out from a certain region, respectively. This study attempts to characterize the similarities and differences between low and high-latitude forests and illustrate the importance of low-latitude forests on the geochemical cycle of POPs. These tasks are conducted by analyzing changes in PCB-153 net atmospheric flow under different forest scenarios and exploring their driving factors.

\section{Method}

\subsection{Chinese Gridded Pesticide Emission and Residue Model with vegetation (ChnGPERM-veg)}

ChnGPERM is a level IV fugacity transport and transfer model for POPs, with a spatial and temporal resolution of $1 / 4^{\circ} \times 1 / 4^{\circ}$ and 1 day, respectively (Tian et al., 2011). This model uses a Lagrangian approach to solve the spatial mass exchange of the target compounds in the low troposphere, the planetary boundary layer, and the atmospheric surface layer. The inter-compartmental transfers of chemicals between the atmospheric surface layer and the ground, including four types of soil, water, and sediment, are calculated on the basis of a level IV fugacity approach. The model has been used in long-term simulations of pesticides and PCBs in China and Asia. The model performance demonstrated in previous studies ensures its usefulness in modeling the environmental fate of these pollutants. In this study, ChnGPERM was on-line coupled with an ecophysiological module of a forest that considers plant leaf physiology, litter formation and decomposition. On the basis of an improved PCB-153 inventory defined on the same spatial and temporal resolution (Xu et al., 2018), the long-term fate of PCB-153 in China's forest from 1965 to 2019 was estimated by ChnGPERM-veg.

\subsection{Forest module}

In the previous version of ChnGPERM, the forest compartment only considered forest soil. The new forest module describes canopy, litter and forest soil compartments. Plant properties and ecophysiological processes are important factors influencing the air-plant exchange of POPs (Barber et al., 2004). Drawing from a physiological canopy model developed by Leuning et al. (1995), Nizzetto and Perlinger (2012) simulated the canopy-air exchange of PCBs by using the leaf temperature and boundary-layer conductance/resistance from an ecophysiological model as the input of a chemical fate model. They demonstrated the possibility to introduce ecophysiological processes into models of the environmental fate of POPs, and illustrated the relevance of processes linked to solar irradiance with dynamics relevant for sub-daily timescales (i.e., stomata opening and leaf heat and water budgets). However, the 
multi-layered ecophysiological model is so complex that on-line coupling it into dynamic regional vegetation model or a long-term regional fate model of POPs would be extremely inefficient. Wang and Leuning (1998) provided an alternative two-leaf model that is least ten times more computationally efficient. The simulated canopy conductance and leaf temperature by the model agree with those by the multilayered model with a relative difference of $<5 \%$. The two-leaf model was on-line incorporated into GhnPERM by the method presented by Nizzetto and Perlinger (2012). In brief, the two-leaf model calculates the canopy-level temperature, stomatal conductance, boundary conductance due to forced convection, and boundary conductance due to free convection for water vapor based on photosynthesis and energy budget. The conductance was corrected by chemical-specific molecular diffusivities and used to generate the overall air-canopy conductance. To be consistent with the level IV fugacity method of GhnPERM (Tian et al., 2011), the mass balance in the air-canopy-litter system was also described by level IV fugacity method (Mackay et al., 2006):

$d\left(f_{c} V_{c} Z_{c}\right) / d t=\left(D_{d_{-} c}+D_{c a}\right) f_{\text {air }}-\left(D_{c a}+D_{l i t}+D_{r_{c}}\right) f_{c}$,

where $f_{c}$ and $f_{\text {air }}$ are fugacity (Pa) of canopy and surface air, respectively; $V_{c}$ is volume $\left(\mathrm{m}^{3}\right) ; Z_{c}$ is fugacity capacity $\left(\mathrm{mol} \mathrm{m}^{-3} \mathrm{~Pa}^{-1}\right)$ of canopy; $D_{d_{-} c}, D_{c a}, D_{\text {lit }}$ and $D_{r_{-} c}$ are the transport coefficients $\left(\mathrm{mol} \mathrm{Pa}^{-1} \mathrm{~d}^{-1}\right)$ of atmospheric dry and wet deposition, air-canopy exchange, litter fall, and degradation in the canopy. Time varying $D_{c a}$ was calculated by the previous method of Nizzetto and Perlinger (2012) considering air-leaf cuticle exchange by advection and air-leaf internal reservoir exchange via stomatal conductance (Section 3.1 of SI). Leaf temperature generated by forest module was used to calculate temperature-dependent fugacity capacity, degradation, physicochemical properties (i.e., $\mathrm{K}_{\mathrm{OA}}$ and Henry's law constant), and diffusivity of heat, air, $\mathrm{CO}_{2}$, and $\mathrm{PCB}-153$. We provided the analytical solution of the air-canopy exchange process and solved other differential equations in the air-litter-soil system by the fifth-order Runge-Kutta method at each time step for simulation efficiency (SI 1.2). This mechanism reduces the model run time and the potential uncertainties associated with the numerical solution of differential equations. Calculation of degradation and foliage-associated deposition fluxes was also presented in SI. The temperature-dependent degradation rates of PCB-153 in the air, water, soil, and sediment were previously described (Xu et al., 2018). The degradation rates in fresh leaf surface and reservoir were assumed to be on the order of magnitude of those in the air and two orders of magnitude longer, respectively (Nizzetto and Perlinger, 2012). The temperature of fallen leaves was assumed to be equal to soil temperature; thus, the degradation rate is the same as that in the soil. The PCB-153 deposition fluxes associated with litter fall were calculated as foliage-associated deposition in this study given that the air-canopy exchange fluxes of previous model include a portion of PCB-153 in the plants that may re-emitted into the air or consumed by degradation (Nizzetto and Perlinger, 2012).

Besides the canopy layer, litter (L) and decomposed litter (DL) layers were added in GhnPERM-veg. Similar to soils, L and DL were assumed to be time-varying layers composed of organic matter $(\mathrm{OM})$, porous air, and water. Therefore, the diffusion, leaching, and degradation rate of PCB-153 in L and DL followed the level IV scheme in the soil of GhnPERM, which was introduced by Jury et al. (1983) and Harner et al. (2001). The OM mass balance in the L and DL layers was determined by the litter fall rate and OM decomposition following the scheme of soil organic matter model (SOMPROF) (Braakhekke et al., 2011). Fig. S2 illustrates the transport processes between air-leaflitter-soil compartments. Details on the forest module construction and parameterization can be found in SI. Other plant compartments, such as root and stem, were not considered in the present model due to the complexity of the coupling a forest module into a regional model, such as GhnPERM. Their contribution to the overall air-toground fluxes for highly hydrophobic compounds, such as PCB-153, is also minor compared with leaf uptake and litter fall (Terzaghi et al.,
2017). Ignoring those factors can improve the efficiency of the GhnPERM-veg program.

The model domain, temperature-dependent physicochemical properties of PCB-153, and model evaluations at non-forest areas have been documented in a previous study (Xu et al., 2018). In this study, the GhnPERM-veg model was driven by meteorological data (1965-2019) obtained from the United States National Center for Environmental Prediction (NCEP) reanalysis dataset (Kalnay et al., 1996). The time-varying leaf area index (LAI) data were from reprocessed MODIS LAI products (Yuan et al., 2011). Four major forests distributed from tropical and cold temperate zone, including evergreen broad-leaved, deciduous broad-leaved, evergreen needle-leaved, and deciduous needle-leaved trees, were considered, and their leaf and litter traits are listed in Tables S1 and S2.

\subsection{Scenario setup and data analysis}

Simulations with varying forest coverages are presented for one model grid for illustrative reasons (Section 1.7 of SI). Forest distribution is dependent on the meteorological and geographical factors. Relative cold temperature and high soil organic content in forests with deciduous tree are also favorable for air-to-ground deposition of PCB-153 and its storage in soils, thus enhancing FFE. To separate the influence of forest canopy and litter fall from temperature and soil carbon pool on the PCB-153 geochemical cycle, simulations in this example were conducted considering scenarios with and without forest coverage, while maintaining unvaried the other environmental parameters for each tree type (Table S3).

To compare the influence of forests on PCB-153 transport and storage for all China, five model scenarios with different forest coverages were applied maintaining the same meteorological, physicochemical and compound emission data as inputs. Thus, SO is a baseline scenario without forest module and leaf-associated exchange and deposition; $\mathrm{S} 1$ is a scenario with current forest coverage in China, depicting the overall influence of forests on fate and transport of POPs; S2 is a scenario with only temperate and boreal forest coverage in China, but without tropical and subtropical forests; S3 is a scenario with tropical and subtropical forest coverage, but excluding the influence of temperate and boreal forests; S4 is similar to S1, but the degradation rates in the canopy is replaced by the rates in the soil, to assess sensitivity on this parameter.

The atmospheric inflow/outflow between grids (referred to as inflow and outflow in the following text) was used to illustrate the FFE. The inflow/outflow fluxes cannot be easily measured or modeled. The GhnPERM-veg simulated the PCB-153 transport and transfer in environmental compartments, including the air, water, sediment, soil, canopy, and litter, based on the mass balance approach. The mass balance between net atmospheric flow, emission, accumulated degradation in all the compartments, accumulated leaching from the deepest soil layer, and final storage in all the compartments can be described by the previous method (Xu et al., 2012):

$\sum_{1965}^{2019}$ flow $=\sum_{1965}^{2019}$ Emission - Storage $e_{2019}-\sum_{1965}^{2019}$ Degradation $-\sum_{1965}^{2019}$ Leaching

In each model grid, a negative value of $\sum_{1965}^{2019}$ flow represents a net atmospheric input, suggesting PCB-153 accumulation or dissipation due to degradation, leaching, or storage in the final stock or residues. A positive value indicates instead a net volatilization from the grid cell. Low to high emission scenarios of PCB emission inventory have been previously proposed (Breivik et al., 2016; Xu et al., 2018). A low emission scenario of an improved PCB-153 inventory in China (Xu et al., 2018) was applied in this study, which yields a better model performance in this study. Re-arranging Eq. (2), total emissions should be 
equal to the sum of storage, degradation, soil leaching, and atmospheric outflow. The accumulated error of calculator floating-point numbers arithmetic operation and numerical solver, such as Runge-Kutta method, may influence the mass conservation in Eq. (2). In this study, the ratio of the sum of residue, degradation, soil leaching, and atmospheric outflow to total emissions was $<7.3 \%$, suggesting a good mass conservation of coding.

The differences between scenarios with and without forests were compared to illustrate the influences of forest:

$R_{x}=m_{x}-m_{0}$

where $m_{0}$ refer to scenario S0 and indicates the value of the parameters included in Eq. (2), and $m_{x}$ indicates the respective values for any of the scenarios with forest (S1 to S4).

\subsection{Model evaluation}

The measured PCB-153 concentrations in soil, sediment, and air samples have been used to validate the model performance of GhnPERM in the previous study (Xu et al., 2018). The modeled soil PCB-153 concentrations correlated well with field data yielding Pearson ( $\mathrm{R}$ ) correlation coefficients of 0.7 between observations and predictions. However, experimental datasets were mainly available for urban, rural or remote sites without vegetation coverage. The concentrations in organic and mineral soil samples from forests in 30 mountains sites across China (Zheng et al., 2014) were used for model conditional validation to evaluate the effect of leaf-litter system on the long-term fate of PCB-153.

In S0, the coefficient for the correlation between the modeled and the observed concentrations in forested soils was 0.22 (Fig. S7A). Without the forest module, the model performance in forests was poor. By contrast, the coefficients between the measured concentrations and modeled results in S1 increased to 0.41 and $0.52(p<0.01)$ for the A and $O$ layer soil samples, respectively (Figs. S7B and S8). This notion suggests that the GhnPERM-veg considerably improved the model performance in forest areas, which can be used to capture the general trends of PCB-153 distribution in China's forests. The performance for forested soils was relatively low compared with the good performance of the modeled for non-forested areas. A possible explanation is that the sub-grid scale spatial variability is more pronounced in forests given that most of those them are distributed in mountainous regions in China. The meteorological, geographical condition, and soil types are spatially more variable in these forest sites than for low land cultivated or residential/industrial areas. The measured concentrations at the same model grid were observed to be highly heterogeneous (Zheng et al., 2014). GhnPERM-veg still cannot capture the corresponding PCB differences at spatial scales that fully capture this variability. Many field observations indicated inter- and intra-specific differences on forest filter efficiencies under the similar environmental and meteorological conditions (Luo et al., 2019; Nizzetto et al., 2008). Site-specific plant traits, such as forest type, leaf thickness, wax layer thickness, LAI, and SLA, can improve the model accuracy. These improvements could not be directly implemented in this version of the model and will be a matter of future developments as soon as relevant data become available to inform better model parameterization.

\section{Results and discussion}

\subsection{Filter effect of different trees}

The previous indicators, such as Arctic contamination potential ( $\mathrm{Su}$ and Wania, 2005), are difficult to use in quantifying FFEs in a regional model. The PCB-153 outflow reduction ratio (Eq. s32) could be used to demonstrate the filter effect in this study. When the air mass passes through a model grid with canopy, PCB-153 can enter the ground surface by litter fall, atmospheric dry and wet deposition, or air-ground exchange, and reenter the air via surface-air volatilization. After depositing on the canopy and soil, the contaminant can further accumulate in soil and vegetation or be lost to the water environment by leaching and runoff (Fig. S2). Terzaghi et al. (2017) displayed the "dispenser" effect of canopy and litter fall by discussing the temporal reversible deposition and evaporation of PCBs in a forest system. In our illustrative simulation (Table S3), the accumulated net downward fluxes in forest scenarios (SBE1, SBD1, SNE1, and SND1) were 1.45 to 2.88 times larger than those in non-forest scenarios (SBE0, SBD0, SNE0, and SND0). Meanwhile, a fraction of PCB-153 was retained (storage) or degraded in the air and canopy compartments, and the remaining PCB-153 was transported out by air mass movement (outflow). The outflow reduction ratio (Eq s32) essentially reflected the total fraction of POPs lost by degradation and leaching or stored in a grid.

The PCB-153 air flow reduction percentages were 19.8\%, 20.2\%, $26.0 \%$, and $21.8 \%$ in forests with broad-leaved evergreen trees (SBE1), broad-leaved deciduous trees (SBD1), needle-leaved evergreen trees (SNE1), and needle-leaved deciduous trees (SND1), respectively. The needle-leaved tree distributed in a relatively cold region exhibited a relatively high reduction rate. The four tree types increased approximately $11.7 \%$ to $13.9 \%$ PCB-153 consumption or accumulation in a grid compared with the rates of $7.79 \%, 6.32 \%, 14.3 \%$, and $9.26 \%$ in non-forest scenarios (SBE0, SBD0, SNE0, and SND0). This notion suggests that all the forest types distributed from tropical regions (broad-leaved evergreen tree) to cold regions (needle-leaved deciduous tree) are capable of influencing the regional cycle of PCB-153. The long-term simulations (S0 to S4) were conducted in China to specify the role of different forests on the PCB-153 cycle in spatially explicit regional environment.

\subsection{PCB-153 atmospheric flow pattern in China}

Fig. S9 compares the spatial distribution of PCB-153 net flow when forests are present ( $\mathrm{S} 1$ ) or not (S0). The red areas representing the net atmospheric outflow of PCB-153 are distributed in the urbanized and densely populated southeast part of Mainland China, which was mainly controlled by high density of primary sources in the east (Fig. S1). The blue areas around the red areas denote the grids with net inflow. As a congener with relatively low vapor pressure, the atmospheric transport of PCB-153 is delayed by frequent condensation events. The blue areas are generally located close to the sources indicating effective scavenging by forest canopies and other environmental compartments. The range of blue areas in S1 was relatively smaller than that in S0. Such a net decline in areas of net inflow indicates that forests retard the spread of PCB-153.

In figures excluding grids without forest coverage (Fig. S10), the boreal forest in Northeast China enhanced the inflow and possibly formed a PCB-153 sink there. Meanwhile, approximately 30\% of the forest areas characterized by net atmospheric outflow in S0 (Fig. S10A) turned into a net inflow pattern in S1 (Fig. S10B), especially in source regions, such as South and Southwest China. GhnPERM and GhnPERM-veg allow the coexistence of the five land cover types in one model grid cell (Tian et al., 2011). The FFE operated by a forested land cover in a given grid may offset net volatilization prevailing in other land-cover areas with the same grid. In summary, the presence of forests either increased the net inflow or reduced the net outflow of PCB-153, consistent with the widely accepted notion that forests effectively increase air-to-ground deposition of POPs (McLachlan and Horstmann, 1998). An exception to this pattern is the reduction of net deposition flux (refers to the sum of all the air-toground depositions in the following) in areas downwind forested region (SI 2.2). However, this behavior further confirms the strong influence of forest in altering budgets of POPs on a regional level. To better display the spatial trend, the difference in net flow calculated in S0 (Fig. S10A) from that in S1 (Fig. S10B) is plotted in Fig. 1. Forests increased the inflow in the southeast of Mainland China with the highest enhancement in areas close to sources (Dark blue region in Fig. 1). Fig. 1 shows that most forested areas in China could reduce the net atmospheric outflow 

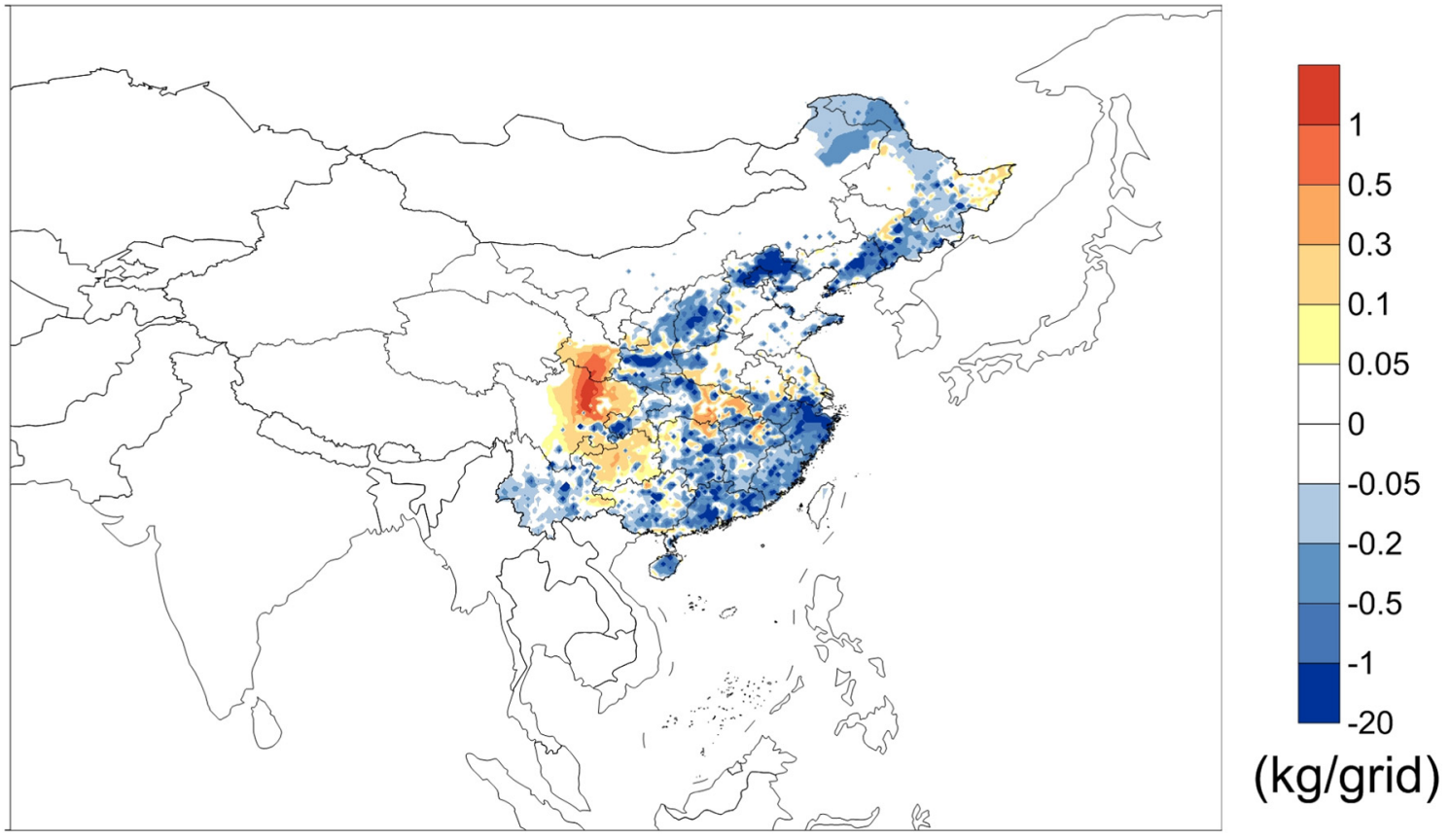

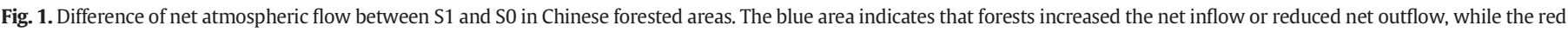
area suggests an opposite effect.

of PCB-153, including those located in low-latitude warm regions. In a previous study focusing on $\alpha-\mathrm{HCH}$, net inflow regions characterized by the three requisites of relatively high organic content, high atmospheric dry and wet deposition, and low temperature were found to be favorable for the retention of POPs (Xu et al., 2013). High-latitude forests widely considered as a global sink of POPs perfectly fit all three prerequisites. By contrast, low-latitude forests did not fit the temperature requisite. Most sink regions of global significance were characterized by low temperature, such as the polar region (Ma et al., 2016), high latitude soils (Dalla Valle et al., 2005), alpine areas (Gong et al., 2019) or boreal forests (Moeckel et al., 2009). To date, the environmental processes in tropical and subtropical forests were still not fully included in the conceptual frame of global POP fate and distribution (Zheng et al., 2015). Limited studies mainly focused on the leaching processes. The reduction of PCB-153 outflow induced by low-latitude forests implies that those forests may also exert an influence on a regional or broader cycle of POPs. Characterizing processing of POPs by low-latitude forests and beyond from an empirical perspective will be necessary to further elucidate this role.

\subsection{Characteristics of forests in China}

Given that the emission scenario was maintained constant in the model simulation, PCB-153 inflow/outflow masses are determined by three processes including storage, degradation, and leaching (Eq. (2)). The outflow reduction or inflow increases must be related to increases of masses in residue, degradation, and leaching categories. Mass changes before and after considering forests in the three categories were compared to illustrate the influences of forests.

\subsubsection{Leaching}

Leaching has been highlighted as a process enhancing the mobility of PCBs in forests (Zheng et al., 2015). The total leaching mass in S1 was 3.18 times that of S0, which is consistent with elevated leaching rates observed in field studies (Zheng et al., 2015). The differences of the accumulated leaching in S1 and S0 are plotted in Fig. S12. High enhancement of leaching mainly occurred in heavily weathered forest in the South China with high dissolved organic matters and rainfall.
However, leaching contributed a minor proportion to the overall mass balance due to the low water-solubility of PCB-153 (Fig. S13). The contribution of leaching to the overall PCB-153 mass in storage, degradation, and leaching categories ranged from $0.10 \%$ in S0 to $0.33 \%$ in $\mathrm{S} 1$. Even if the total leaching amount in scenarios with forests (S1 to S4) was higher than that in S0, it is still a negligible portion on a regional scale.

\subsubsection{Storage}

PCB-153 is predicted to be mainly stored in the soil compartment because of its large volume and fugacity capacity. Forests can accumulate POPs and efficiently transfer them to the soil. The soil organic matter can possibly act as a final sink or temporary repository of POPs (Terzaghi et al., 2017). In S0, forest soil without canopy contributed $31.9 \%$ to the national PCB-153 stored residue. The percentages of the total residue of PCB-153 stored in forest soil, litter, and canopy were $33.5 \%, 2.6 \%$ and $<0.01 \%$ in S1 (Fig. S14), respectively. After the forest compartments are introduced in S1, the residue in forests, including forest soil, canopy, and litter, was 1.15 times that in S0. Fig. S15 illustrates that the PCB-153 residues in S1 were higher than those in S0 in the areas with forest coverage, especially those closed to eastern sources. The storage in forests increased to the detriment of the reduced stored residue in grids in the north of those forested areas. Residues in other compartments declined to $95.2 \%$ compared with that in S0. Such residues are widely distributed in central and Northeast China (blue areas in Fig. S15). In summary, the environmental residues in Mainland China of S1 became 1.01 times that of S0 (Table S7).

The low and high-latitude forests increased the PCB-153 residues in forested areas but reduced the exposure and accumulation of contaminants in the storage of adjacent areas, especially those located on the north of forests (given the dominant wind direction in this region). In S2, a significant residue reduction area in the northeast extended to Russia (Fig. 2A). In combination with the residue enhancement in forest, the total residue in China exhibited a minor increase trend that is 1.01 times that in SO (Table S7). By contrast, the residue reduction areas in S3 were still within China (Fig. 2B). Low-latitude forests mainly moved the PCB-153 from its north to those forests. The total residue in the country was still similar to that in S0 (Table S7), which means 


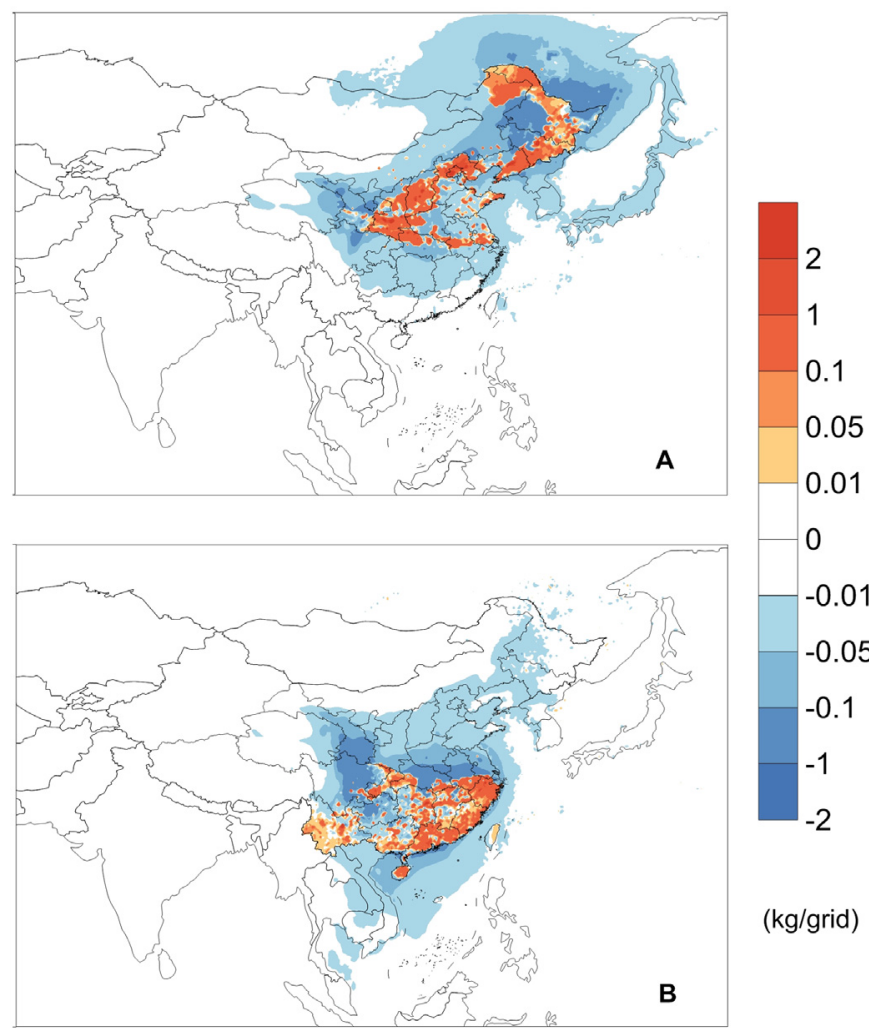

Fig. 2. Differences of PCB-153 residues between S2 and S0 (A), and between S3 and S0 (B), representing the influences of high-latitude and low-latitude forests.

that low-latitude forests did not influence the overall residues of PCB153 in China.

\subsubsection{Degradation}

Degradation is an important process controlling dissipation and the overall mass balance of PCB-153 (Fig. S13). Forests can enhance the movement of POPs from media with fast degradation, such as the air, to those with longer degradation half-lives, such as litter or soil ( $\mathrm{Su}$ and Wania, 2005). Accordingly, the overall degradation in S1 should be lower than that in S0, unless some fast degradation processes occurred. The total degradation flux in forested grids in S1 was 1.18 times that in S0. Meanwhile, the masses of PCB-153 in other areas were $96.5 \%$ compared with those in S0. Fig. S16 plots the overall degradation changes from $\mathrm{S} 0$ to $\mathrm{S} 1$. The increase in degradation fluxes can be observed in forest areas; however, the reduction in non-forest areas is not as significant as Fig. S15. The overall degradation of PCB-153 was 1.03 times that of S0 (Table S7). This value is equivalent to an extra $1.37 \%$ of emitted PCB-153 consumed by degradation in S1.

Fast degradation in the forest canopy has been suggested as a reason for enhanced degradation in forests (Maddalena et al., 2003; Su and Wania, 2005). Following the degradation scheme of Nizzetto and Perlinger (2012), the degradation rate in plants in this study was assumed to be lower than that in the air but higher than that in the soil. We replaced the degradations rate within the canopy by the rate in the soils to test whether degradation in canopy enhanced the overall degradation of PCB-153 in China (S4). The degradation flux is still higher than that in S0 (Table S7), even though fast degradation in canopy was excluded. This notion indicates that the degradation enhancement related to forests cannot be simply related to the fast degradation rate in the forest canopy alone.

Degradation rates were only assumed to be varied with temperature in the model. Other factors, such as sunlight exposure inducing a high level of antioxidants in the leaves (Bussotti, 2008), have not been considered yet. Therefore, the enhanced degradation in S1 should be related to a relatively high temperature in southern forested areas (Fig. S17) and the increased FFE operated by the canopies that drove to higher accumulation in the soil of these forests. Forests slowed down the northward movement of PCB-153 impoverishing the residue stored in colder Northern latitude soils. The higher PCB-153 residue stored in warmer soil drove the overall higher degradation loss calculated in S1 compared with S0. A mass center analysis was performed to explore the relative location of PCB-153. The barycenter is the point where all the masses are perfectly balanced. This factor was calculated to illustrate the overall trend of pollutant distribution and transport trends (Xu et al., 2012). The calculated mass centers of PCB-153 are plotted in Fig. 3. The barycenter of PCB-153 moved from East to West China from 1965 to

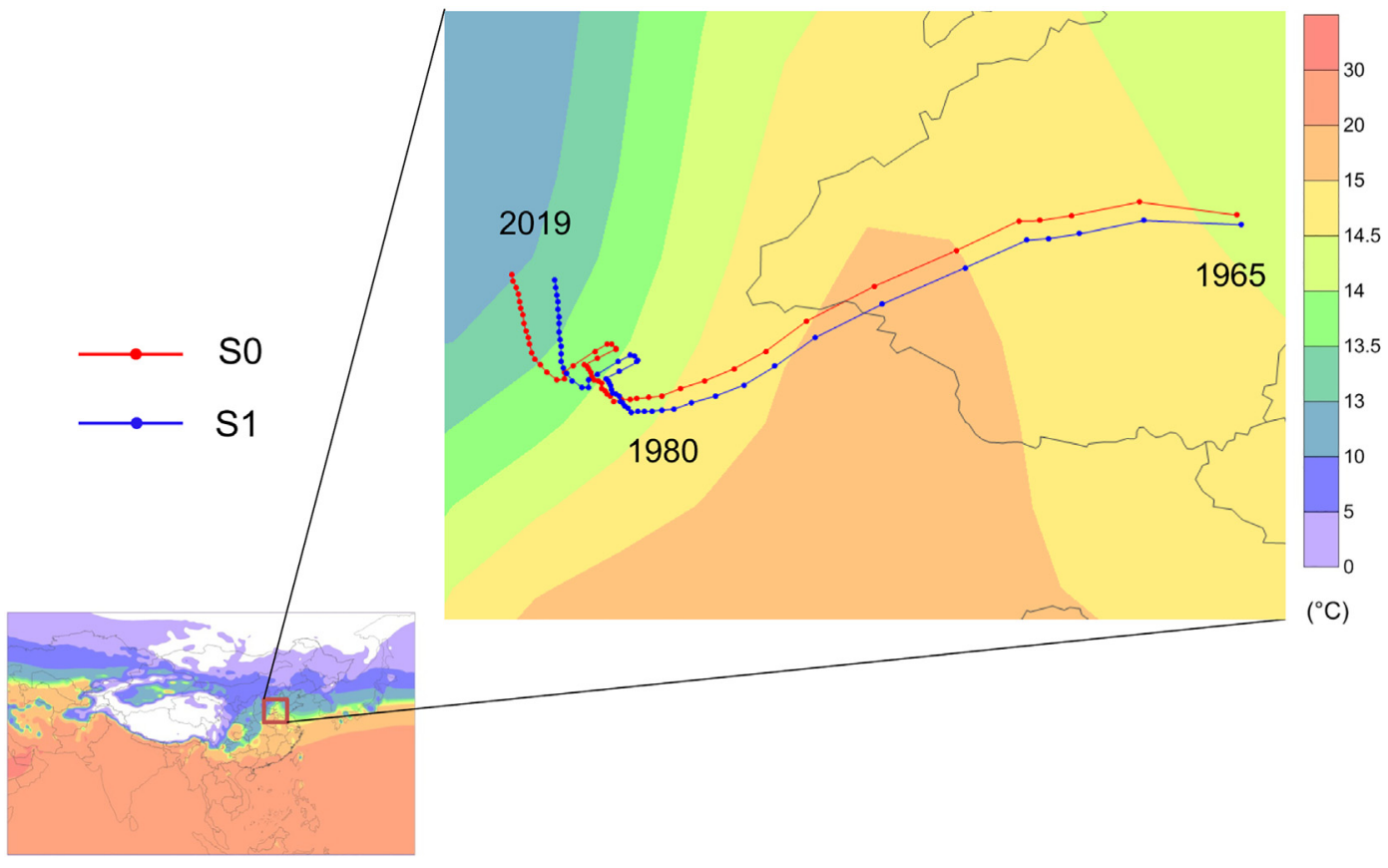

Fig. 3. Mass center movement path in S0 (red line) and S1 (blue line) from 1965 to 2009; the dots indicate the location of barycenter in each year. 
1980, following the spread of PCB-containing products application during that period. In the 1980s, the first batch of PCB-containing products in East China began to be disposed; therefore, the barycenter moved back towards the east. After 2005, environmental processes controlled the movement of PCB-153 when the primary emission started to decline. Similar to other POPs, the barycenter gradually moved towards the north. During the whole process of the PCB-153 movement, the mass center in $\mathrm{S} 1$ was located to the southeast of the mass center in S0, meaning that a larger portion of PCB-153 distributed in the southeast areas of that in S0. Southeast China was characterized by higher temperature. Therefore, a higher degradation should be higher in S1.

The low and high-latitude forests influenced the degradation fluxes (Fig. 4), with increased degradation in forested areas. The total degradation amounts in S2 and S3 were 1.06 and 1.05 times those in S0, suggesting that the atmospheric outflow reduction in the two forest groups can be linked to enhanced degradation. As previously discussed, the influence of low-latitude forests on the overall residues of PCB-153 in China was minor. Degradation is the major process in reducing the PCB-153 outflow in low-latitude forests. The contribution to the total sink exceeded $50 \%$ in the southeast of China (Fig. S18). In high-latitude forests, degradation and residue contributed to the PCB-153 atmospheric outflow reduction with residue as the major one (Fig. S18).

\subsection{National PCB-153 outflow and lifetime in China}

According to the ratios of PCB-153 masses stored in the modeled environment, degradation and leaching fluxes in relation to the total emission (Fig. S13), storage and degradation are the major processes. Small perturbation in the two categories can easily increase or decrease the atmospheric inflow or outflow. Previous model simulation proved that FFE can effectively reduce long-range atmospheric transport of POPs by comparing indicators, such as characteristic travel distance or Arctic

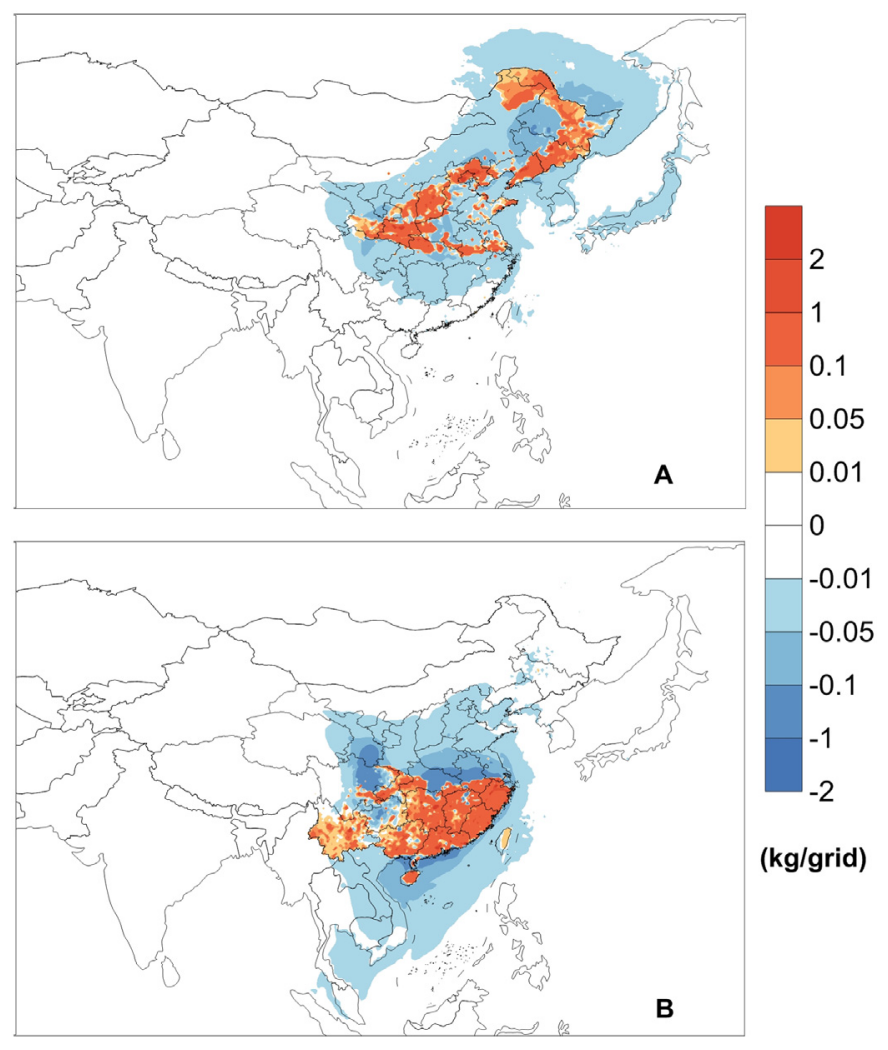

Fig. 4. Differences of accumulated degradation masses between S2 and S0 (A), and between S3 and S0 (B). contamination potential (Maddalena et al., 2003; Su and Wania, 2005). The overall outflow mass of PCB-153 from mainland China was calculated to explore the extent to which FFE influences the atmospheric transport in a spatially explicit regional environment. The atmospheric outflow mass in S1 was $69 \%$ of that in S0 (Table S7). As a congener with relatively low mobility, the outflow of PCB-153 accounted for a small fraction of total emission, and its contribution decreased from $6.7 \%$ to $4.6 \%$ as an effect of the presence of forest. In the scenario only considering lowlatitude forest (S2) and high-latitude forests (S3), the atmospheric outflow rates were $89 \%$ and $80 \%$ of that in S0. The two forest groups accounted for $67 \%$ and $33 \%$ of the total forest area in China. However, the outflow reduction of high-latitude forests was higher. As suggested in many simulation and observation studies, high-latitude forests are still the most important compartment for reducing the long-range transport of PCB-153 to the north (Moeckel et al., 2009; Su and Wania, 2005). However, the outflow reduction rate of $11 \%$ suggests that the contribution of low-latitude forest cannot be ignored in the estimation.

The outflow reduction of POPs is achieved at the expense of longer environmental residence time (Moeckel et al., 2009). Following the previous definition of the overall global lifetime of PCBs, we define the lifetime of a substance in China as the ratio of the total emission amount to the overall loss rate by outflow, degradation, and leaching in Mainland China. The calculated lifetimes were 104.2, 105.1, 105.3, and 104.0 years in S0, S1, S2, and S3, respectively. In comparison with the lifetime of several decades in the global model Globo-POP (Wania and Daly, 2002), the relatively longer lifetime in this study could be attributed to the thicker soil layer of GhnPERM (Tian et al., 2011) and high emission into the soil in China (Xu et al., 2018). In previous model estimations, vegetation compartment always increased the lifetime or persistence of POPs in the environment (Su and Wania, 2005; Wegmann et al., 2004). Similar patterns were found in forests scenario (S1) and high-latitude forest scenario only (S2). By contrast, the inclusion of tropical and subtropical forests (S3) decreased the simulated overall lifetime of PCB-153 in China. Accumulation in tropical and subtropical forests with high temperature and heavy precipitation inevitably increases loss rates due to degradation in soil and leaching. Given that approximately $52 \%$ of global forests are in the tropical and subtropical areas (Keenan et al., 2015), the lifetime reduction further highlights the special roles of low-latitude forests on the global cycle of POPs.

\section{Conclusion}

This study provided a preliminary dynamic model estimation of PCB153 distributions in China's forest. The presence of forests can reduce the atmospheric outflow from China by $31 \%$ via increasing leaching, degradation, and residues following sequestration of airborne PCB-153 by forest canopies and its transfer to soil. The FFE in temperate and boreal forests can be related to increased residues and degradation. Meanwhile the FFE in tropical and subtropical forests can be linked to increased degradation. Although the FFE of low-latitude forests is relatively weak, it could still contribute to the outflow and lifetime reduction of PCB-153 in China and should not be neglected in regional mass budgets. Considering the rapid forest growth and the threat from global warming, the influence of low-latitude forests could become prominent in the future.

\section{CRediT authorship contribution statement}

Yue Xu: Conceptualization, Methodology, Software, data collection and validation, Writing - original draft, Formal analysis.

Chongguo Tian: Conceptualization, Methodology, Software, Writing original draft, Writing - review\& editing.

Luca Nizzetto: Methodology, Software, Writing - review \& editing, Supervision.

Gan Zhang: Conceptualization, Writing - review \& editing, Supervision. 


\section{Declaration of competing interest}

The authors declare that they have no known competing financial interests or personal relationships that could have appeared to influence the work reported in this paper.

\section{Acknowledgements}

The authors appreciate the financial support from the National Natural Science Foundation of China (NSFC) (nos.: 41673133, 41877401 and 71403118), National Key Research and Development Program of China (ATMSYC, 2017YFC0212000), and the Strategic Priority Research Programs of the Chinese Academy of Sciences, the Pan-Third Pole Environment Study for a Green Silk Road (Pan-TPE, XDA2004050201).

\section{Appendix A. Supplementary data}

Supplementary data to this article can be found online at https://doi. org/10.1016/j.scitotenv.2021.146285.

\section{References}

Barber, J.L., Thomas, G.O., Kerstiens, G., Jones, K.C., 2004. Current issues and uncertainties in the measurement and modelling of air-vegetation exchange and within-plant processing of POPs. Environ. Pollut. 128, 99-138.

Braakhekke, M.C., Beer, C., Hoosbeek, M.R., Reichstein, M., Kruijt, B., Schrumpf, M., Kabat, P., 2011. SOMPROF: a vertically explicit soil organic matter model. Ecol. Model. 222, 1712-1730.

Breivik, K., Armitage, J.M., Wania, F., Sweetman, A.J., Jones, K.C., 2016. Tracking the global distribution of persistent organic pollutants accounting for e-waste exports to developing regions. Environ. Sci. Technol. 50, 798-805.

Bussotti, F., 2008. Functional leaf traits, plant communities and acclimation processes in relation to oxidative stress in trees: a critical overview. Glob. Change Biol. 14, 2727-2739.

Dalla Valle, M., Jurado, E., Dachs, J., Sweetman, A.J., Jones, K.C., 2005. The maximum reservoir capacity of soils for persistent organic pollutants: implications for global cycling. Environ. Pollut. 134, 153-164.

Dong, N., Prentice, I.C., Harrison, S.P., Song, Q.H., Zhang, Y.P., 2017. Biophysical homoeostasis of leaf temperature: a neglected process for vegetation and landsurface modelling. Glob. Ecol. Biogeogr. 26, 998-1007.

Gong, P., Wang, X., Pokhrel, B., Wang, H., Liu, X., Liu, X., Wania, F., 2019. Trans-Himalayan transport of organochlorine compounds: three-year observations and model-based flux estimation. Environ. Sci. Technol. 53, 6773-6783.

Harner, T., Bidleman, T.F., Jantunen, L.M.M., Mackay, D., 2001. Soil-air exchange model of persistent pesticides in the United States cotton belt. Environ. Toxicol. Chem. 20, $1612-1621$.

Horstmann, M., McLachlan, M.S., 1998. Atmospheric deposition of semivolatile organic compounds to two forest canopies. Atmos. Environ. 32, 1799-1809.

Huang, T., Zhang, X., Ling, Z., Zhang, L., Gao, H., Tian, C., Guo, J., Zhao, Y., Wang, L., Ma, J., 2016. Impacts of large-scale land-use change on the uptake of polycyclic aromatic hydrocarbons in the artificial three northern regions shelter forest across northern China. Environ. Sci. Technol. 50, 12885-12893.

Jiang, H., Apps, M.J., Zhang, Y., Peng, C., Woodard, P.M., 1999. Modelling the spatial pattern of net primary productivity in Chinese forests. Ecol. Model. 122, 275-288.

Jury, W.A., Spencer, W.F, Farmer, W.J., 1983. Behavior assessment model for trace organics in soil: I. Model description1. J. Environ. Qual. 12, 558-564.

Kalnay, E., Kanamitsu, M., Kistler, R., Collins, W., Deaven, D., Gandin, L., Iredell, M., Saha, S., White, G., Woollen, J., Zhu, Y., Chelliah, M., Ebisuzaki, W., Higgins, W., Janowiak, J., Mo, K.C., Ropelewski, C., Wang, J., Leetmaa, A., Reynolds, R., Jenne, R., Joseph, D., 1996. The NCEP/NCAR 40-year reanalysis project. Bull. Amer. Meteorol. Soc. 77, 437-472.

Keenan, R.J., Reams, G.A., Achard, F., de Freitas, J.V., Grainger, A., Lindquist, E., 2015. Dynamics of global forest area: results from the FAO global forest resources assessment 2015. For. Ecol. Manag. 352, 9-20.

Leuning, R., Kelliher, F.M., Depury, D.G.G., Schulze, E.D., 1995. Leaf nitrogen, photosynthesis, conductance and transpiration - scaling from leaves to canopies. Plant Cell Environ. $18,1183-1200$
Liu, X., Li, J., Zheng, Q., Bing, H., Zhang, R., Wang, Y., Luo, C., Liu, X., Wu, Y., Pan, S., Zhang, G., 2014. Forest filter effect versus cold trapping effect on the altitudinal distribution of PCBs: a case study of Mt. Gongga, eastern Tibetan Plateau. Environ. Sci. Technol. 48, 14377-14385.

Liu, X., Wang, S., Jiang, Y., Sun, Y., Li, J., Zhang, G., 2017. Polychlorinated biphenyls and polybrominated diphenylethers in soils from planted forests and adjacent natural forests on a tropical island. Environ. Pollut. 227, 57-63.

Luo, Y., Yang, R., Li, Y., Wang, P., Zhu, Y., Yuan, G., Zhang, Q., Jiang, G., 2019. Accumulation and fate processes of organochlorine pesticides (OCPs) in soil profiles in Mt. Shergyla, Tibetan Plateau: a comparison on different forest types. Chemosphere 231, 571-578.

Ma, J., Hung, H., Macdonald, R.W., 2016. The influence of global climate change on the environmental fate of persistent organic pollutants: a review with emphasis on the northern hemisphere and the Arctic as a receptor. Glob. Planet. Change 146, 89-108.

Mackay, D., Foster, K.L., Patwa, Z., Webster, E., 2006. Chemical partitioning to foliage: the contribution and legacy of Davide Calamari. Environ. Sci. Pollut. Res. Int. 13, 2-8.

Maddalena, R.L., McKone, T.E., Riley, W.J., 2003. Is there a "forest filter effect" for organic pollutants? Stoch. Environ. Res. Risk Assess. 17, 231-234.

McLachlan, M.S., Horstmann, M., 1998. Forests as filters of airborne organic pollutants: a model. Environ. Sci. Technol. 32, 413-420.

Moeckel, C., Nizzetto, L., Strandberg, B., Lindroth, A., Jones, K.C., 2009. Air-boreal forest transfer and processing of polychlorinated biphenyls. Environ. Sci. Technol. 43, 5282-5289.

Nizzetto, L., Perlinger, J.A., 2012. Climatic, biological, and land cover controls on the exchange of gas-phase semivolatile chemical pollutants between forest canopies and the atmosphere. Environ. Sci. Technol. 46, 2699-2707.

Nizzetto, L., Pastore, C., Liu, X., Camporini, P., Stroppiana, D., Herbert, B., Boschetti, M. Zhang, G., Brivio, P.A., Jones, K.C., Di Guardo, A., 2008. Accumulation parameters and seasonal trends for PCBs in temperate and boreal forest plant species. Environ. Sci. Technol. 42, 5911-5916.

Qiu, Z., Feng, Z., Song, Y., Li, M., Zhang, P., 2020. Carbon sequestration potential of forest vegetation in China from 2003 to 2050: predicting forest vegetation growth based on climate and the environment. J. Clean. Prod. 252, 119715.

Su, Y., Wania, F., 2005. Does the forest filter effect prevent semivolatile organic compounds from reaching the Arctic? Environ. Sci. Technol. 39, 7185-7193.

Tang, X., Zhao, X., Bai, Y., Tang, Z., Wang, W., Zhao, Y., Wan, H., Xie, Z., Shi, X., Wu, B. Wang, G., Yan, J., Ma, K., Du, S., Li, S., Han, S., Ma, Y., Hu, H., He, N., Yang, Y., Han, W., He, H., Yu, G., Fang, J., Zhou, G., 2018. Carbon pools in China's terrestrial ecosystems: new estimates based on an intensive field survey. Proc. Natl. Acad. Sci. U. S. A. $115,4021-4026$.

Terzaghi, E., Morselli, M., Semplice, M., Cerabolini, B.E.L., Jones, K.C., Freppaz, M., Di Guardo, A., 2017. SoilPlusVeg: an integrated air-plant-litter-soil model to predict organic chemical fate and recycling in forests. Sci. Total Environ. 595, 169-177.

Tian, C., Liu, L., Ma, J., Tang, J., Li, Y.-F., 2011. Modeling redistribution of $\alpha-\mathrm{HCH}$ in Chinese soil induced by environment factors. Environ. Pollut. 159, 2961-2967.

Wang, Y.P., Leuning, R., 1998. A two-leaf model for canopy conductance, photosynthesis and partitioning of available energy I: model description and comparison with a multi-layered model. Agric. For. Meteorol. 91, 89-111.

Wania, F., Daly, G.L., 2002. Estimating the contribution of degradation in air and deposition to the deep sea to the global loss of PCBs. Atmos. Environ. 36, 5581-5593.

Wegmann, F., Scheringer, M., Möller, M., Hungerbühler, K., 2004. Influence of vegetation on the environmental partitioning of DDT in two global multimedia models. Environ. Sci. Technol. 38, 1505-1512.

Xu, Y., Tian, C., Ma, J., Zhang, G., Li, Y.-F., Ming, L., Li, J., Chen, Y., Tang, J., 2012. Assessing environmental fate of $\beta-\mathrm{HCH}$ in Asian soil and association with environmental factors. Environ. Sci. Technol. 46, 9525-9532.

Xu, Y., Tian, C.G., Zhang, G., Ming, L.L., Wang, Y., Chen, Y.J., Tang, J.H., Li, J., Luo, C.L., 2013. Influence of monsoon system on alpha-HCH fate in Asia: a model study from 1948 to 2008. J. Geophys. Res.-Atmos. 118, 6764-6770.

Xu, Y., Tian, C., Wang, X., Ma, J., Tang, J., Chen, Y., Li, J., Zhang, G., 2018. An improved inventory of polychlorinated biphenyls in China: a case study on PCB-153. Atmos. Environ. 183, 40-48.

Yuan, H., Dai, Y., Xiao, Z., Ji, D., Shangguan, W., 2011. Reprocessing the MODIS Leaf Area Index products for land surface and climate modelling. Remote Sens. Environ. 115, 1171-1187.

Zheng, Q., Nizzetto, L., Mulder, M.D., Sáňka, O., Lammel, G., Li, J., Bing, H., Liu, X., Jiang, Y. Luo, C., Zhang, G., 2014. Does an analysis of polychlorinated biphenyl (PCB) distribution in mountain soils across China reveal a latitudinal fractionation paradox? Environ. Pollut. 195, 115-122.

Zheng, Q., Nizzetto, L., Liu, X., Borgå, K., Starrfelt, J., Li, J., Jiang, Y., Liu, X., Jones, K.C., Zhang, G., 2015. Elevated mobility of persistent organic pollutants in the soil of a tropical rainforest. Environ. Sci. Technol. 49, 4302-4309. 